\title{
Retraction Note to: Decreased expression of the long noncoding RNA LINC00261 indicate poor prognosis in gastric cancer and suppress gastric cancer metastasis by affecting the epithelial-mesenchymal transition
}

Yu Fan ${ }^{1 \dagger}$, Yan-fen Wang ${ }^{2 \dagger}$, Hua-fang Su${ }^{3}$, Na Fang $^{1}$, Chen Zou', Wen-feng Li ${ }^{3}$ and Zheng-hua Fei ${ }^{3 *}$

\section{Retraction}

The authors have retracted this article [1] because of significant overlap of text and some images with a previously published article by $\mathrm{Xu}$ et al. [2]. A formal investigation by the 1st Affiliated Hospital of Wenzhou Medical University has found that some panels in Fig. 6b [1] are identical with panels in Fig. 5a [2] and some images of mice lungs in Fig. 6c [1] are identical with images in Fig. 3g [2]. The data reported in this article are therefore unreliable. All authors agree to this retraction.

\footnotetext{
Author details

${ }^{1}$ Cancer Institute, The Affiliated People's Hospital of Jiangsu University, Zhenjiang, People's Republic of China. ${ }^{2}$ Department of Pathology, the First People's Hospital of Yangzhou/The Second Clinical Medical College, Yangzhou University, Yangzhou, People's Republic of China. ${ }^{3}$ Radiotherapy and Chemotherapy Department, The 1st Affiliated Hospital of Wenzhou Medical University, Wenzhou, China.
}

Received: 13 December 2017 Accepted: 21 December 2017

Published online: 04 January 2018

\section{References \\ 1. Fan Y, Wang Y, Su H, Fang N, Zou C, Li W, Fei Z. Decreased expression of the long noncoding RNA LINC00261 indicate poor prognosis in gastric cancer and suppress gastric cancer metastasis by affecting the epithelial-mesenchymal transition. J Hematol Oncol. 2016;9:57. https://doi.org/10.1186/s13045-016-0288-8. \\ 2. Xu T, Huang M, Xia R, Liu X, Sun M, Yin L, Chen W, Han L, Zhang E, Kong R, De $W$, Shu Y. Decreased expression of the long non-coding RNA FENDRR is associated with poor prognosis in gastric cancer and FENDRR regulates gastric cancer cell metastasis by affecting fibronectin1 expression. J Hematol Oncol. 2014;7:63. https://doi.org/10.1186/s13045-014-0063-7.}

\footnotetext{
* Correspondence: hui-y@163.com

${ }^{\dagger}$ Equal contributors

${ }^{3}$ Radiotherapy and Chemotherapy Department, The 1st Affiliated Hospital of Wenzhou Medical University, Wenzhou, China
} 